\title{
Kink-antikink, trapping bags and five-dimensional Gauss-Bonnet gravity
}

\author{
Massimo Giovannini* \\ Centro “Enrico Fermi", Compendio del Viminale, Via Panisperna 89/A, 00184 Rome, Italy \\ and Department of Physics, Theory Division, CERN, 1211 Geneva 23, Switzerland
}

(Received 19 September 2006; published 25 October 2006)

\begin{abstract}
Five-dimensional Gauss-Bonnet gravity, with one warped extra-dimension, allows classes of solutions where two scalar fields combine either in a kink-antikink system or in a trapping-bag configuration. While the kink-antikink system can be interpreted as a pair of gravitating domain walls with opposite topological charges, the trapping-bag solution consists of a domain wall supplemented by a nontopological defect. In both classes of solutions, for large absolute values of the bulk coordinate (i.e. far from the core of the defects), the geometry is given by five-dimensional anti-de Sitter space.
\end{abstract}

DOI: 10.1103/PhysRevD.74.087505

It is known since the pioneering works of Lanczos [1] (see also [2]) that, in more than four space-time dimensions, the Einstein-Hilbert action can be supplemented by the so-called Euler-Gauss-Bonnet combination (see also [3] for a recent review). Such an inclusion leads to field equations that involve, at most, second derivatives of the metric. The Gauss-Bonnet combination arises also naturally as first correction in the string tension expansion to the low-energy string effective action [4-9].

An apparently unrelated observation is that, in the presence of infinite extra-dimensions, fields of various spin may be localized around higher dimensional defects (see, for instance, [10]). Indeed, in the past few years, various analytical solutions containing gravitating defects have been discussed either in the context of Einstein-Hilbert gravity or in the framework of Brans-Dicke gravity [1116]. Some of these solutions are compatible with fivedimensional anti-de Sitter space-time (in what follows $\mathrm{AdS}_{5}$ ) for large absolute value of the bulk coordinate, providing, in this way a smooth realization of the Randall-Sundrum set-up [17] where the matter content is given by branes (i.e. gravitating kinks) of finite thickness.

The purpose of the present paper is to show that, in Gauss-Bonnet gravity, there exists solutions compatible with $\mathrm{AdS}_{5}$ and containing pairs of gravitating defects rather than a single defect. Solutions have been obtained in the presence of Gauss-Bonnet gravity but only in the case of single defects [18-21].

Pairs of defects are known to exist in $(1+1)$ field theories in flat space-time and in the presence of appropriately nonlinear interaction potentials [22-24]. As a consequence of the intrinsic nonlinearity of the problem, exact solutions are rare even if specific methods have been devised in order to deal with the integration of the systems in rather general terms (see $[23,25]$ and references therein). In the presence of gravity it is more difficult to reduce the problem to the quadrature and to find analytical solutions.

*Electronic address: massimo.giovannini@cern.ch
PACS numbers: 04.50.+h, 05.45.Yv, 11.10.Kk, 11.25.Mj

This difficulty is even more severe in Gauss-Bonnet gravity.

Consider then the case where the gravity part of the action takes the form ${ }^{1}$

$$
S_{\mathrm{g}}=-\int d^{5} x \sqrt{|G|}\left(\frac{R}{2 \kappa}+\alpha^{\prime} \mathcal{R}_{\mathrm{EGB}}^{2}\right)
$$

where $G_{A B}$ is the metric tensor, $R$ is the Ricci scalar and

$$
\mathcal{R}_{\mathrm{EGB}}^{2}=R^{2}-4 R_{A B} R^{A B}+R_{A B C D} R^{A B C D},
$$

is the Euler-Gauss-Bonnet (EGB) combination. In Eq. (1), $\kappa=8 \pi G_{5}=8 \pi / M^{3}$ and $\alpha^{\prime}$ has dimensions of an energy scale, i.e., in natural units, an inverse length.

The matter part of the action includes two scalar degrees of freedom, denoted by $\phi$ and $\chi$, interacting via the potential $W(\phi, \chi)$ :

$$
\begin{aligned}
S_{\mathrm{m}}= & \int d^{5} x \sqrt{|G|}\left[\frac{1}{2} G^{A B} \partial_{A} \phi \partial_{B} \phi+\frac{1}{2} G^{A B} \partial_{A} \chi \partial_{B} \chi\right. \\
& -W(\phi, \chi)],
\end{aligned}
$$

The total action will then be given by the sum of the gravity and matter action, i.e. $S_{\mathrm{t}}=S_{\mathrm{g}}+S_{\mathrm{m}}$. The equations of motion are obtained by taking the functional derivative of $S_{\mathrm{t}}$ with respect to the metric tensor and with respect to the two scalar fields. Functional derivation with respect to the metric tensor leads to the generalized Einstein-Lanczos equations

$$
R_{A}^{B}-\frac{1}{2} \delta_{A}^{B}=\kappa \mathcal{T}_{A}^{B}-2 \alpha^{\prime} \kappa \mathcal{Q}_{A}^{B},
$$

where

\footnotetext{
${ }^{1}$ The signature of the metric is mostly minus, i.e. $(+,-,-,-,-,-)$. Latin (capital) indices run over the fivedimensional space-time; Greek indices run over the $(3+$ 1)-dimensional space-time with Minkowskian signature.
} 


$$
\begin{aligned}
\mathcal{T}_{A}^{B}= & \partial_{A} \phi \partial^{B} \phi+\partial_{A} \chi \partial^{B} \chi-\delta_{A}^{B}\left[\frac{1}{2} G^{M N} \partial_{M} \phi \partial_{N} \phi\right. \\
& \left.+\frac{1}{2} G^{M N} \partial_{M} \chi \partial_{N} \chi-W(\phi, \chi)\right], \\
\mathcal{Q}_{A}^{B}= & \frac{1}{2} \delta_{A}^{B} \mathcal{R}_{\mathrm{EGB}}^{2}-2 R R_{A}^{B}+4 R_{A C} R^{C B}+4 R_{C D} R_{A}{ }^{C B D} \\
& -2 R_{A C D E} R^{B C D E},
\end{aligned}
$$

are, respectively, the energy-momentum tensor and the Lanczos tensor. Functional derivation with respect to $\phi$ and $\chi$ produces the following pair of Klein-Gordon equations:

$$
G^{A B} \nabla_{A} \nabla_{B} \phi+\frac{\partial W}{\partial \phi}=0, \quad G^{A B} \nabla_{A} \nabla_{B} \chi+\frac{\partial W}{\partial \chi}=0,
$$

where, we recall, $\nabla_{A} \nabla_{B}=\partial_{A} \partial_{B}-\Gamma_{A B}^{C} \partial_{C}$ when applied to a scalar degree of freedom.

In the case of a five-dimensional warped metric of the type characterized by a bulk coordinate $w$, i.e.

$$
d s^{2}=a^{2}(w)\left[\eta_{\mu \nu} d x^{\mu} d x^{\nu}-d w^{2}\right]
$$

Denoting with the prime a derivation with respect to $w$, the explicit form of Eq. (4) becomes:

$$
\begin{gathered}
\mathcal{H}^{\prime}\left(1-\frac{2 \epsilon \mathcal{H}^{2}}{a^{2}}\right)+\mathcal{H}^{2}\left(1-\frac{2 \epsilon \mathcal{H}^{\prime}}{a^{2}}\right) \\
=-\frac{\kappa}{3}\left[\frac{\phi^{\prime 2}}{2}+\frac{\chi^{\prime 2}}{2}+a^{2} W(\phi, \chi)\right], \\
\mathcal{H}^{2}\left(1-\frac{2 \epsilon \mathcal{H}^{2}}{a^{2}}\right)=\frac{\kappa}{6}\left[\frac{\phi^{\prime 2}}{2}+\frac{\chi^{\prime 2}}{2}-a^{2} W(\phi, \chi)\right],
\end{gathered}
$$

where $\mathcal{H}=(\ln a)^{\prime}$. In Eqs. (9) and (10) the quantity $\epsilon=$ $2 \kappa \alpha^{\prime}$ has been also defined and it has dimensions, in natural units, of a length squared. Using Eq. (8) into Eqs. (7) the following explicit equations are obtained:

$$
\begin{aligned}
& \phi^{\prime \prime}+3 \mathcal{H} \phi^{\prime}-a^{2} \frac{\partial W}{\partial \phi}=0, \\
& \chi^{\prime \prime}+3 \mathcal{H} \chi^{\prime}-a^{2} \frac{\partial W}{\partial \chi}=0 .
\end{aligned}
$$

By combining Eqs. (9) and (10), the explicit from of the Einstein-Lanczos equations can be also written as

$$
\begin{aligned}
& \left(\phi^{\prime 2}+\chi^{\prime 2}\right)=\frac{3}{\kappa}\left(\mathcal{H}^{2}-\mathcal{H}^{\prime}\right)\left[1-\frac{4 \epsilon}{a^{2}} \mathcal{H}^{2}\right], \\
& W=-\frac{3}{2 a^{2} \kappa}\left\{\left(\mathcal{H}^{2}+\mathcal{H}^{\prime}\right)\left[1-\frac{4 \epsilon}{a^{2}} \mathcal{H}^{2}\right]+2 \mathcal{H}^{2}\right\} .
\end{aligned}
$$

Consider then the situation where the warp factor tends to $\mathrm{AdS}_{5}$ for large absolute value of the bulk coordinate $w$. A possible choice of warp factor with the desired proper- ties is

$$
\begin{gathered}
a(w)=\frac{a_{0}}{\sqrt{b^{2} w^{2}+1}}, \quad \mathcal{H}=-\frac{b^{2} w}{b^{2} w^{2}+1}, \\
\mathcal{H}^{\prime}=\frac{b^{2}\left(b^{2} w^{2}-1\right)}{\left(b^{2} w^{2}+1\right)^{2}},
\end{gathered}
$$

where $a_{0}$ is a free parameter that will be determined from the compatibility with the whole system of equations. In Eq. (14) the first relation is the ansatz for the warp factor while the remaining relations follow from the definition of $\mathcal{H}$ in terms of $a(w)$.

The method employed in order to find the solution is constructive in the sense that we impose the geometry given in Eq. (14) and then get the solution by satisfying the Einstein-Lanczos equations as well as the KleinGordon equations. In particular, using Eq. (14), it is not difficult to show that Eqs. (12) and (13) imply, respectively,

$$
\begin{gathered}
\phi^{\prime 2}+\chi^{\prime 2}=\frac{3 b^{2}}{\kappa} \frac{1}{\left(b^{2} w^{2}+1\right)^{3}}, \\
W(\phi, \chi)=-\frac{3}{8 \kappa \epsilon} \frac{2 b^{4} w^{4}+4 b^{2} w^{2}-1}{\left(b^{2} w^{2}+1\right)^{2}} .
\end{gathered}
$$

From Eq. (15) we deduce that $\phi$ and $\chi$ are given by

$$
\begin{aligned}
& \phi(w)=\frac{v}{\sqrt{2}}\left(1+\frac{b w}{\sqrt{b^{2} w^{2}+1}}\right)^{3 / 2}, \\
& \chi(w)=\frac{v}{\sqrt{2}}\left(1-\frac{b w}{\sqrt{b^{2} w^{2}+1}}\right)^{3 / 2} .
\end{aligned}
$$

provided the arbitrary constants $a_{0}$ and $v$ are such that

$$
a_{0}=2 \sqrt{\epsilon} b, \quad v^{2}=\frac{4}{3 \kappa} \text {. }
$$

The first of these two relations is necessary in order to write Eqs. (15) and (16) while the second relation is essential to solve Eq. (15) in terms of Eqs. (17) and (18). Knowing the form of the field profiles, the potential can be determined from Eq. (16) by adopting the following ansatz:

$$
\begin{aligned}
W(\phi, \chi)= & \mathcal{A}\left(\phi^{2}+\chi^{2}\right)^{2}+\mathcal{B}\left(\phi^{2}+\chi^{2}\right)+\mathcal{C} \\
& +\mathcal{L}(\phi, \chi) .
\end{aligned}
$$

The functional $\mathcal{L}(\phi, \chi)$ vanishes exactly on the classical solution given by Eqs. (17) and (18) but its derivatives do contribute to the Klein-Gordon equations. In fact Eq. (11) can then be used to determine $\mathcal{L}(\phi, \chi)$. Using Eqs. (17) and (18) into Eq. (20) and recalling Eq. (16), the coefficients appearing in Eq. (20) are determined to be

$$
\begin{gathered}
\mathcal{A}=\frac{1}{8 \kappa \epsilon v^{4}}=\frac{3}{32 v^{2}} \quad \mathcal{B}=-\frac{1}{\kappa \epsilon v^{2}}=-\frac{3}{4 \epsilon}, \\
\mathcal{C}=\frac{5}{4 \kappa \epsilon}=\frac{15}{16}\left(\frac{v^{2}}{\epsilon}\right),
\end{gathered}
$$

where the second equality in each of the three relations follows by eliminating $\kappa$ according to Eq. (19) (second 
equality). Inserting then Eqs. (14), (17), (18), and (20) into Eq. (11), the functional form of $\mathcal{L}(\tilde{\phi}, \tilde{\chi})$ can be determined:

$$
\mathcal{L}(\tilde{\phi}, \tilde{\chi})=\frac{7}{2} \frac{v^{2}}{\epsilon}\left(|\tilde{\phi}|^{2 / 3}+|\tilde{\chi}|^{2 / 3}-1\right)\left(1-\tilde{\phi}^{2}-\tilde{\chi}^{2}\right)^{2},
$$

where, for notational convenience, we defined the two rescaled fields $\tilde{\phi}=\phi /(2 v)$ and $\tilde{\chi}=\chi /(2 v)$. Using the second relation in Eq. (19) into Eq. (21) to eliminate $\kappa$ in favor of $v^{2}$, the complete form of the potential becomes, in terms of $\tilde{\phi}$ and $\tilde{\chi}$,

$$
\begin{aligned}
W(\tilde{\phi}, \tilde{\chi})= & \frac{3 v^{2}}{2 \epsilon}\left(\tilde{\phi}^{2}+\tilde{\chi}^{2}\right)^{2}-\frac{3 v^{2}}{\epsilon}\left(\tilde{\phi}^{2}+\tilde{\chi}^{2}\right)+\frac{15}{16} \frac{v^{2}}{\epsilon} \\
& +\frac{7}{2} \frac{v^{2}}{\epsilon}\left[|\tilde{\phi}|^{2 / 3}+|\tilde{\chi}|^{2 / 3}-1\right]\left[1-\tilde{\phi}^{2}-\tilde{\chi}^{2}\right]^{2} .
\end{aligned}
$$

The solution given in Eqs. (17) and (18) implies, necessarily, that $\phi>0$ and $\chi>0$. However, it appears from the analytical form of the potential that also $\phi \rightarrow-\phi$ or $\chi \rightarrow$ $-\chi$ lead to acceptable solutions and this is the rationale for the absolute values in Eqs. (22) and (23). In Fig. 1 (plot at the left hand side) the kink-antikink solution of Eqs. (17) and (18) is reported as a function of the rescaled bulk radius $b w$. In the case of one spatial dimension, spatial infinity consists of two points, i.e. $\pm \infty$; a topological charge is then customarily defined for the characterization of $(1+1)$-dimensional defects such as the ones arising in the case of sine-Gordon system [22]. In the case of the kink-antikink system the topological charges can be defined as

$$
Q_{\phi}=\frac{1}{2 \pi} \int_{-\infty}^{\infty} \frac{\partial \phi}{\partial w} d w, \quad Q_{\chi}=\frac{1}{2 \pi} \int_{-\infty}^{\infty} \frac{\partial \chi}{\partial w} d w .
$$

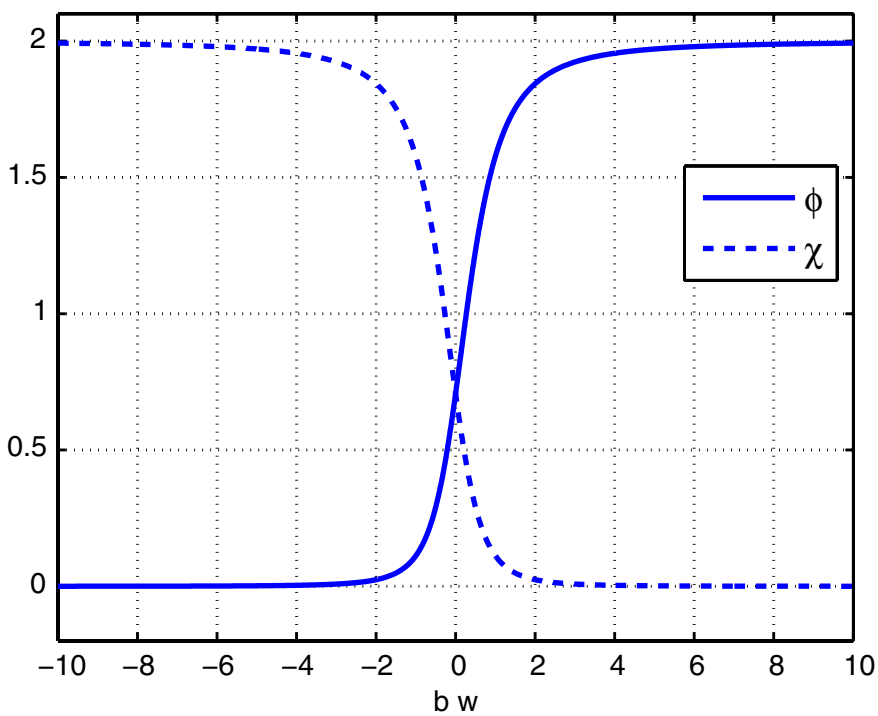

Inserting the explicit solutions of Eqs. (17) and (18) into Eq. (24) it is easy to find that $Q_{\phi}=-Q_{\chi}=v / \pi$.

By slightly modifying the form of the potential obtained in the case of the kink-antikink system one can obtain solutions of a different kind. In $(1+1)$ dimensions these solutions are known as trapping-bag solutions. Exactly with the same procedure described above it can be shown that the following field profiles

$$
\begin{aligned}
\phi(w)= & \frac{v}{2 \sqrt{2}}\left[\left(1+\frac{b w}{\sqrt{b^{2} w^{2}+1}}\right)^{3 / 2}\right. \\
& \left.+\left(1-\frac{b w}{\sqrt{b^{2} w^{2}+1}}\right)^{3 / 2}\right], \\
\chi(w)= & \frac{v}{2 \sqrt{2}}\left[\left(1+\frac{b w}{\sqrt{b^{2} w^{2}+1}}\right)^{3 / 2}\right. \\
& \left.-\left(1-\frac{b w}{\sqrt{b^{2} w^{2}+1}}\right)^{3 / 2}\right],
\end{aligned}
$$

are solutions of the evolution equations previously deduced for the following choice of the potential

$$
\begin{aligned}
W(\phi, \chi)= & \frac{3 v^{2}}{\epsilon}\left(\tilde{\phi}^{2}+\tilde{\chi}^{2}\right)^{2}-\frac{3 v^{2}}{\epsilon}\left(\tilde{\phi}^{2}+\tilde{\chi}^{2}\right)+\frac{15 v^{2}}{32 \epsilon} \\
& +\frac{7 v^{2}}{\epsilon}\left(|\tilde{\phi}+\tilde{\chi}|^{2 / 3}+|\tilde{\phi}-\tilde{\chi}|^{2 / 3}-1\right) \\
& \times\left[\frac{1}{2}-\tilde{\phi}^{2}-\tilde{\chi}^{2}\right]^{2} .
\end{aligned}
$$

In this case the Einstein-Lanczos equations, i.e. Eqs. (9) and (10), are satisfied only if

$$
a_{0}=2 \sqrt{\epsilon} b, \quad v^{2}=\frac{8}{3 \kappa},
$$

i.e. the $a_{0}$ is the same as in Eq. (19) while the relation of $v^{2}$ to $\kappa$ is different. It is clear that Eqs. (25) and (26) look like being the sum and the difference of the two profiles dis-

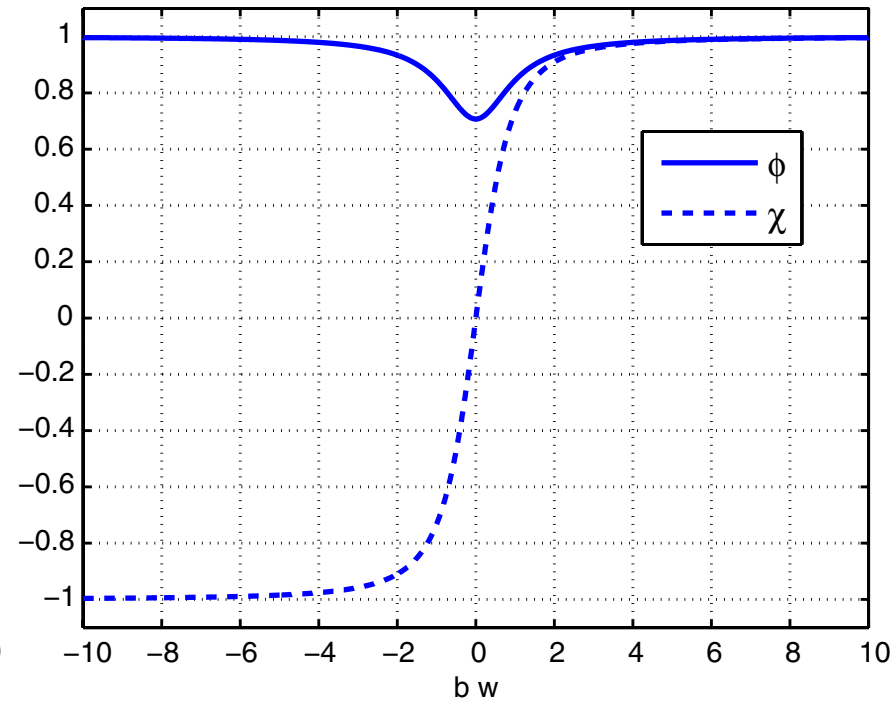

FIG. 1 (color online). The kink-antikink solution (left plot) and the trapping-bag solution (right plot) are illustrated as a function of the bulk radius. 
cussed above in Eqs. (17) and (18). The system under consideration is, however, intrinsically nonlinear and, therefore, the compatibility of the solutions (25) and (26) entails necessarily a different relation between $v^{2}$ and $\kappa$ (compare Eqs. (19) and (28)) and also a slightly different form of the potential.

In Fig. 1 (plot at the right) the analytical solution of Eqs. (25) and (26) is illustrated for $v=1$. From Fig. 1 it is also clear the rationale for the terminology employed in naming these solutions. The $\phi$ field is the "bag" that "traps" the $\chi$ field. As already mentioned this type of trapping-bag solutions can be found, in $(1+1)$ dimensions, and with an appropriate nonlinear potential possessing a global $U(1)$ symmetry [23-25]. By inserting Eqs. (25) and (26) into Eq. (24), it is easy to show that while $Q_{\phi}=0, Q_{\chi}=v / \pi$. So, while the $\phi$ field illustrates a nontopological profile, the $\chi$ field is still topological.

The constructive technique exploited in the present paper can be extended in other cases when, for instance, the form of the underlying geometry is different from the one of Eq. (14). In particular, it might be interesting to discuss the warp factor

$$
a(w)=a_{1}\left[(b w)^{2 \nu}+1\right]^{-1 / 2 \nu},
$$

where $\nu \geq 1$ is an integer parameter. Notice that for $\nu=1$ Eq. (29) gives exactly Eq. (14). For $\nu>1 \mathrm{AdS}_{5}$ is always recovered, asymptotically, for large absolute value of the bulk radius. Single field defects (both topological and non- topological) arising in the geometry (29) have been analyzed in [26] in the case of Einstein-Hilbert gravity. It would be interesting to generalize these solutions to the case of Einstein-Lanczos gravity and in the presence of a pair of scalar degrees of freedom. Along similar lines it seems also reasonable to think about the possibility of multidefects, i.e. gravtating profiles of two (or more) scalar degrees of freedom.

In the present investigation it has been argued that there may be a nontrivial interplay between five-dimensional Gauss-Bonnet gravity and the presence of unusual defects that may arise when two scalar degree of freedom are simultaneously present. Solutions describing both kinkantikink profiles and trapping bags have been presented. While the possibility of qalitatively similar profiles in nonlinear $(1+1)$ dimensional field theories has been established in a number of different ways, in the context of five-dimensional Gauss-Bonnet gravity no attention has been payed to these configurations, to the best of our knowledge. One of the interesting features of the obtained solutions is that the geometry which solves the EinsteinLanczos equations in the presence either of kink-antikink profiles or in the presence of trapping-bag profiles is always of $\mathrm{AdS}_{5}$. More specifically the warp factor tends to $\mathrm{AdS}_{5}$ for $|w| \rightarrow \infty$. Close to the core of the defect, i.e. for $|w| \rightarrow 0$ the geometry is always regular (i.e. all curvature invariants are regular); both $\phi$ and $\chi$ (as well as $\phi^{\prime}$ and $\chi^{\prime}$ ) are finite and regular.
[1] C. Lanczos, Z. Phys. 73, 147 (1932); Ann. Math. 39, 842 (1938).

[2] D. Lovelock, J. Math. Phys. (N.Y.) 12, 498 (1971).

[3] N. Deruelle and J. Madore, gr-qc/0305004.

[4] J. Madore, Phys. Lett. A 110, 289 (1985); 111, 283 (1985).

[5] B. Zwiebach, Phys. Lett. B 156, 315 (1985).

[6] D. G. Boulware and S. Deser, Phys. Rev. Lett. 55, 2656 (1985); Phys. Lett. B 175, 409 (1986).

[7] R. R. Metsaev and A. A. Tseytlin, Phys. Lett. B 191, 354 (1987); Nucl. Phys. B293, 385 (1987).

[8] C. G. Callan, E. J. Martinec, M. J. Perry, and D. Friedan, Nucl. Phys. B262, 593 (1985).

[9] A. Sen, Phys. Rev. Lett. 55, 1846 (1985).

[10] V. A. Rubakov, Usp. Fiz. Nauk 171, 913 (2001) [Phys. Usp. 44, 871 (2001)].

[11] A. Kehagias and K. Tamvakis, Phys. Lett. B 504, 38 (2001); Mod. Phys. Lett. A 17, 1767 (2002); C. Bogdanos, A. Dimitriadis, and K. Tamvakis, Phys. Rev. D 74, 045003 (2006).

[12] M. Gremm, Phys. Lett. B 478, 434 (2000); Phys. Rev. D 62, 044017 (2000).

[13] M. Giovannini, Phys. Rev. D 64, 064023 (2001); Class. Quant. Grav. 20, 1063 (2003).

[14] R. Koley and S. Kar, Class. Quant. Grav. 22, 753 (2005);
S. Pal and S. Kar, Class. Quant. Grav. 23, 2571 (2006).

[15] S. Randjbar-Daemi and M. Shaposhnikov, Nucl. Phys. B645, 188 (2002); S. Randjbar-Daemi, Mod. Phys. Lett. A 18, 2459 (2003).

[16] K. Farakos and P. Pasipoularides, Phys. Lett. B 621, 224 (2005); hep-th/0609089.

[17] L. Randall and R. Sundrum, Phys. Rev. Lett. 83, 3370 (1999); 83, 4690 (1999).

[18] M. Giovannini, Phys. Rev. D 63, 064011 (2001); 64, 124004 (2001).

[19] O. Corradini and Z. Kakushadze, Phys. Lett. B 494, 302 (2000).

[20] N. Deruelle and C. Germani, Nuovo Cimento Soc. Ital. Fis. B 118, 977 (2003).

[21] N.E. Mavromatos and J. Rizos, Phys. Rev. D 62, 124004 (2000).

[22] R. Rajaraman, Solitons and Instantons (Elsevier, Amsterdam, 1982).

[23] R. Rajaraman and E. Weinberg, Phys. Rev. D 11, 2950 (1975).

[24] C. Montonen, Nucl. Phys. B112, 349 (1976).

[25] A. de Souza Doutra, Phys. Lett. B 626, 249 (2005).

[26] M. Giovannini, CERN-PH-TH-2006-150. 